\title{
Meteor Studies
}

\author{
B. A. Lindblad \\ Box 43, S-221 00 Lund Sweden
}

Historically meteor astronomy is one area where amateurs have always been able to make significant contributions. In fact, in the 19th century, it was amateur naked eye and telescopic observations which laid down much of the foundations of meteor astronomy. References to this work can be found in any textbook on meteors. The 19th century observers concentrated on counting meteors, estimating magnitudes and plotting the meteor paths on star maps. Their main interest was to determine hourly rates and shower radiants. An important milestone was Denning's radiant catalogue (Denning 1882), which included 4367 shower radiants. Although it is now believed that many of these radiants are spurious, the catalogue is still a useful reference. Unfortunately Denning and other 19 th century observers often combined sporadic meteors observed on different nights into a minor stream radiant. This habit of "radiant hunting" is even today quite popular among some amateur observers. However, in all fairness it should be emphasized that most of the 20th century amateur meteor observers applied very strict criteria to their radiant determinations. Names such as J.M. Prentice in Great Britain, R.A. McIntosh in New Zealand and R. Rigollet in France may be mentioned.

The extensive development of professional meteor programs after the second World War involving photographic, radar and image orthicon techniques added vastly to our knowledge of meteors and thereby no doubt caused a temporary decline in amateur activities. Fortunately there is today a new generation of amateur observers who work very professionally. This new generation observes meteors by classical techniques, but in addition it uses electronic devices such as tape recorders, personal computers and forward scatter radio systems, thereby successfully competing with the professional scientists. Today there is a high degree of cooperation between the amateur observers. A number of amateur meteor societies and their publications are listed in Table 1. The listed societies have existed for a very long time - in one case for more than 50 years. The Dutch and Belgian reports are referenced in Astronomy and Astrophysics Abstracts.

In what way can today's amateur observers contribute to meteor astronomy? The answer is obvious: The professional astronomer has neither time nor money to maintain a constant sky watch. In the 1950's and 1960's a number of meteor radars kept a 


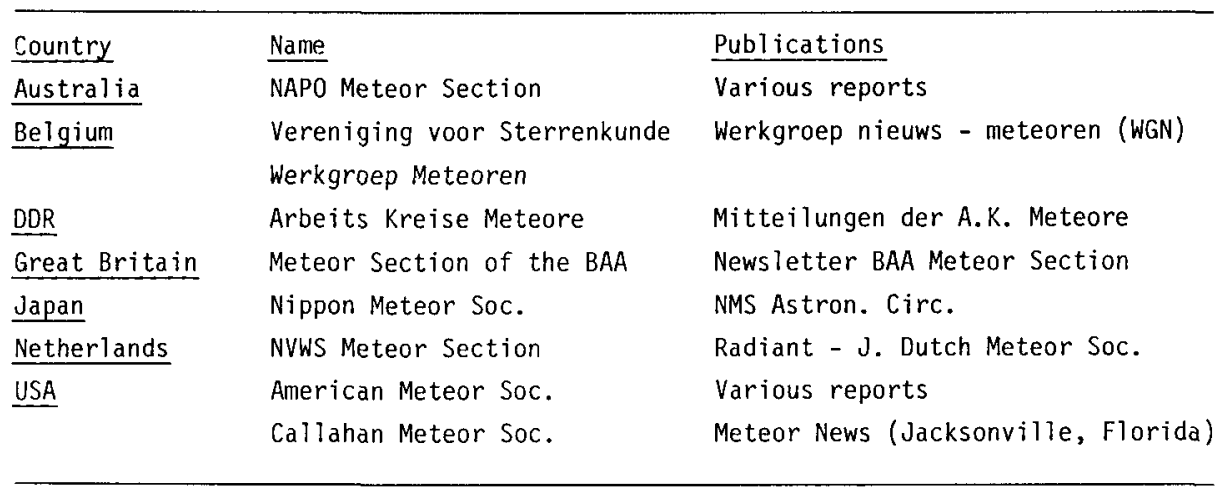

semi-permanent watch on meteor activity. Unfortunately, most of these programs were terminated in the 1960's and 1970's. The demise of the meteor radars in most western countries means that any new meteor shower which suddenly appears in the night sky will pass undetected, unless it is detected by an amateur group.

In this talk I will highlight three cases where amateur observers recently have made significant contributions to meteor astronomy.

\section{Observations of the Sigma Puppid stream associated with comet Grigg-Skjellerup}

In $1964 \mathrm{P} /$ Grigg-Skjellerup made a close approach to Jupiter. The Jupiter encounter perturbed the comet's orbit, so that from 1967 and on very close approaches to the Earth's orbit occur. Sitarski (1964) predicted that a meteor shower would be observed on April 23 in 1967, 1972 and 1977. The theoretical radiant of this stream is $\alpha=109^{\circ}, \delta=-45^{\circ}$. The far southern declination of the radiant implies that only a limited number of amateur groups are available for observing this meteor shower. Since the stream is newly born, total shower duration may be only a few hours. Thus the shower may be observed only over a limited part of the Earth. In 1967 the comet-Earth distance at the nodal point was only 0.0027 a.u., but the Earth crossed the nodal line 97 days later than the comet. It was doubtful if fresh cometary debris had spread that far along the orbit.

No Sigma Puppid shower was observed in 1967 and 1972 indicating that the cometary debris was still in close proximity of the comet. In 1977 an intense, shortlived Sigma Puppid shower was observed by Australian amateurs (Wood 1986). Continued visual observations of this shower in 1982-84 by the same group indicated that the cometary material was gradually spreading along the orbit and in the orbital plane of the comet. For, perhaps, the first time we can study the birth and subsequent evolution of a meteoroid stream. The Australian amateur observations are our only source of information on this unique meteor stream. A review of these observations is given in Lindblad (1986a). 
A Japanese amateur, M. Koseki, has compiled a list of all photographic two station meteor orbits published in the scientific literature and searched this sample on a micro-computer for meteor streams (Koseki 1986). Koseki has also checked the original orbits and found a substantial number of errors in the published orbital elements! Koseki has kindly supplied the IAU meteor data center in Lund with a list of these errors. This is a very impressive piece of work done by the new computer generation of amateur astronomers.

\section{Observations of the Giacobinid meteor stream by forward scatter techniques}

Forward scatter radio techniques for the study of meteors is gaining increasing popularity amongst amateur groups. The technique is in principle quite simple - you listen to the carrier frequency of a TV station at some $1000 \mathrm{kms}$ distance and record meteor burst signals on a tape recorder. However, the geometry of meteor forward scatter is quite complicated, and interpretation of the data demands considerble skill. For a discussion of the observability function of forward meteor scatter see Steyaert (1987) where additional references can be found.

During the 1985 recurrence of the Giacobinid meteor shower (associated with comet Giacobini-Zinner) several amateurs made radio recordings of the intense, short duration Giacobinid display. A British amateur Mason (1986) has studied the hourly meteor burst rate versus time. Mason's hourly rate curve agrees closely with a curve obtained by Lindblad (1986b) using the Onsala meteor radar. The time of peak activity agreed to within $1 \mathrm{~min}$.

\section{$\underline{\text { References }}$}

Koseki, M., 1986, Brit. Astron. Assoc, , 96, pp. 232-240.

Lindblad, B.A., 1986a, ESA SP-250, vol. III, pp. 399-400.

LindbTad, B.A., 1986b, ESA SP-250, vol. I1, pp. 229-231.

Mason, J., 1986, ESA SP-250, vol. III, pp. 379-384.

Sitarski, G., 1964, Acta Astronomica, 14, pp. 25-37.

Steyaert, C., 1987, Werkgroep nieuws - Meteoren, 15, pp. 90-93.

Wood, J., 1986, priv. comm. See also Western Australian Meteor Soc. Bu11., 80. 